\section{ANNOUNCEMENTS}

\section{Minisymposium}

der Arbeitsgemeinschaft Schwingungsspektroskopie an Polymeren

vom 29. 9. bis 1. 10. 1982 an der Universität Ulm

Das Minisymposium dient dem Erfahrungsaustausch von Wissenschaftlern aus Hochschule und Industrie, die sich mit der Schwingungsspektroskopie - insbesondere an Polymeren und Modellsystemen - beschäftigen.

Das Treffen bietet die Möglichkeit sowohl experimentelle und theoretische Forschungsergebnisse als auch methodische und apparative Neuentwicklungen zur Diskussion zu stellen.

Dr. H. P. Grossmann

Abteilung Angewandte Physik

Universität Ulm

D-7900 Ulm $\cdot$ Oberer Eselsberg

Tel. : (0731) $1762476 / 2290$

(Kontaktadresse)

\section{JOINT MEETING}

Organized by the Institute of Colloid and Surface Science at Clarkson College

June 24-28, 1985

59th Colloid and Surface Science Symposium

sponsored by

Division of Colloid and Surface Chemistry of

American Chemical Society

and

\section{5th International Conference on Surface and Colloid Science \\ sponsored by \\ International Association of Colloid and Surface Scientists}

For further information, contact Professor Egon Matijević, Chairman, Department of Chemistry, Clarkson College of Technology, Potsdam, New York 13676. Telephone 315-268-2367/2392.

Am 25. März 1982 verstarb im Alter von 78 Jahren

\title{
Professor Dr. Hans Erbring
}

Uber zwei Jahrzehnte lang hat er als Leiter die Chemische Forschung unseres Hauses erfolgreich geführt. Aufgrund seines reichen Wissens und der großen Erfahrung hat er die wissenschaftliche Entwicklung unseres Hauses entscheidend mitgeprägt.

Wir trauern um eine Persönlichkeit, mit der uns über die Arbeit hinaus eine tiefe Freundschaft verband. Obwohl Herr Professor Erbring schon vor einigen Jahren in den Ruhestand trat, war und wird die Erinnerung an ihn immer lebendig bleiben.

DR. MADAUS \& CO.

Geschäftsleitung und Belegschaft

Köln, im März 1982 